\title{
Adolescent and young adult brain tumors: current topics and review
}

\author{
Fumiyuki Yamasaki ${ }^{1}$ (D)
}

Received: 2 October 2021 / Accepted: 10 November 2021 / Published online: 22 January 2022

(c) The Author(s) under exclusive licence to Japan Society of Clinical Oncology 2021

\begin{abstract}
The management of brain tumors developed in adolescents and young adults (AYAs) is challenging because of their histological heterogeneity and low incidence. The brain tumor and its treatment interventions can negatively affect neurological, neurocognitive, and endocrinological function, and dramatically affect the circumstances of AYA patients progressing to further education, employment, and marriage. Specific support is thus necessary to maintain the quality of life (QOL) of AYA brain tumor patients. AYA patients and survivors require active intervention and support for returning to school or work, progressing to further education, finding employment, and preserving fertility. Recent cancer genome profiling revealed that AYA gliomas include pediatric- and adult-type genetic alteration. Insights into the biology underlying the distribution of tumors in AYAs may influence the development of prospective trials. A more individualized view of brain tumors may influence stratification of patients' in future clinical studies as well as selection for molecular targeted therapy. Here I review strategies for achieving a better outcome to decrease late effects and improve QOL.
\end{abstract}

Keywords Adolescent and young adults $\cdot$ Brain tumor $\cdot$ Late effect $\cdot$ Fertility preservation $\cdot$ Integrated diagnosis

\section{Introduction}

Adolescents and young adults (AYAs) comprise a group aged between 15 and 29-39 years. Here in line with other studies, I define AYAs as aged between 15 and 39 years [1-3]. Malignant neoplasms are the leading cause of death among AYAs, excluding suicide and unexpected accidents [4]. Oncological care of AYA patients is typically not uniform as they frequently fall between the pediatric and adult services in many countries-including Japan-leading to a poor entry into clinical trials, a poorer survival, and many psychosocial issues for AYA patients [5]. Despite recent significant advances in neuro-oncology, brain tumors among AYAs remain a major contributor to morbidity and mortality [5]. Although mortality rates among AYAs with cancer as a whole have declined by approximately $0.8 \%$ per year, the survival rate of AYAs with CNS tumors has not improved [6]. Tumor biology and clinical outcome for a given tumor differ among age groups, suggesting age-specific approaches.

Fumiyuki Yamasaki

fyama@hiroshima-u.ac.jp

1 Department of Neurosurgery, Hiroshima University Hospital, 1-2-3 Kasumi, Minami-ku, Hiroshima, Hiroshima 734-8551, Japan
Brain tumors arising in AYAs are classified as pediatricor adult-type [7]. The period from childhood to adulthood entails great physical, psychological, emotional, social, and sexual change $[8,9]$. Many AYAs with cancer have unmet needs during cancer treatment and beyond, which result in worse emotional functioning, work or school functioning, fatigue, health-related quality of life (QOL) [10]. Moreover, AYAs with brain tumors have additional issues including cognitive impairment and deterioration, hormonal deficiency and deterioration, and appearance change. However, there is a paucity of literature about these problems in AYAs with brain tumors. Care is divided into adult and pediatric care. Changes in behavioral and endocrine function in AYAs complicate the differential diagnosis of brain tumors, often delaying the diagnosis [4]. Here I review the current concerns surrounding AYA brain tumors and their survivors.

\section{Epidemiology of AYA brain tumors}

The spectrum of AYA brain tumors-particularly with respect to location-differs significantly from that of younger children or older adults [5]. Although brain tumors can be observed at any age, brain development and the age of brain tumor incidence appear to be interrelated [11]. Choroid plexus tumor and atypical teratoid/rhabdoid tumor incidence 
peak at infancy. Ependymoma incidence peaks at age 0-4, and medulloblastoma and pilocytic astrocytoma incidence peak at age 5-9. Craniopharyngioma first peaks at age 0-14. Germ cell tumors peak at age 10-19-the timing of testis and ovary development. Pituitary adenoma starts increasing at age 15-19-at activation of pituitary gland hormone secretion-thereafter gradually increasing. Among pituitary adenoma, null cell adenoma and growth hormone-secreting adenoma peak at age 55-59, while prolactinoma peaks at age 25-29. Major adult tumors of meningioma and schwannoma increase from age 30 to 39, while lymphoma is rare in AYAs. Grade III glioma-including anaplastic oligodendroglioma, anaplastic oligoastrocytoma and anaplastic astrocytomapeak at age $30-39$.

In Fig. 1, I show the registered number of primary brain tumors by age group at the brain tumors registry in Japan. Gliomas-including unclassified, neuronal, and glioneuronal tumors - are the most common tumors in all AYAs, followed by germ cell tumors at age 10-19, and pituitary adenomas at age 20-39. Overall, representative malignant brain tumors in AYAs are diffuse gliomas and germ cell tumors.

\section{Breaking bad news in AYA malignant brain tumors}

One of the most important—and difficult-communication situations for both patients with brain tumor and physicians is receiving and giving bad news $[12,13]$. No reports have focused on breaking bad news in AYA malignant brain tumors. AYA patients, especially adolescents, would be disappointed and/or confused about their prospects for further education, employment, independence from parents, and marriage. To communicate bad news sensitively, physicians need to be aware of the possible worst news and the patients' communication preference [14]. Receiving the initial tumor diagnosis was reportedly considered the worst of all bad news for most patients [15]. Some parents desire to hide the name of disease, and the terms "cancer" and "anticancer agent," because they anticipate the stress these terms will give patients. AYAs are familiar with the internet and readily seek information about their own disease. Patients generally wanted to know "the truth" about diagnosis and prognosis, but what they meant varied; not all patients appear to wish to know the

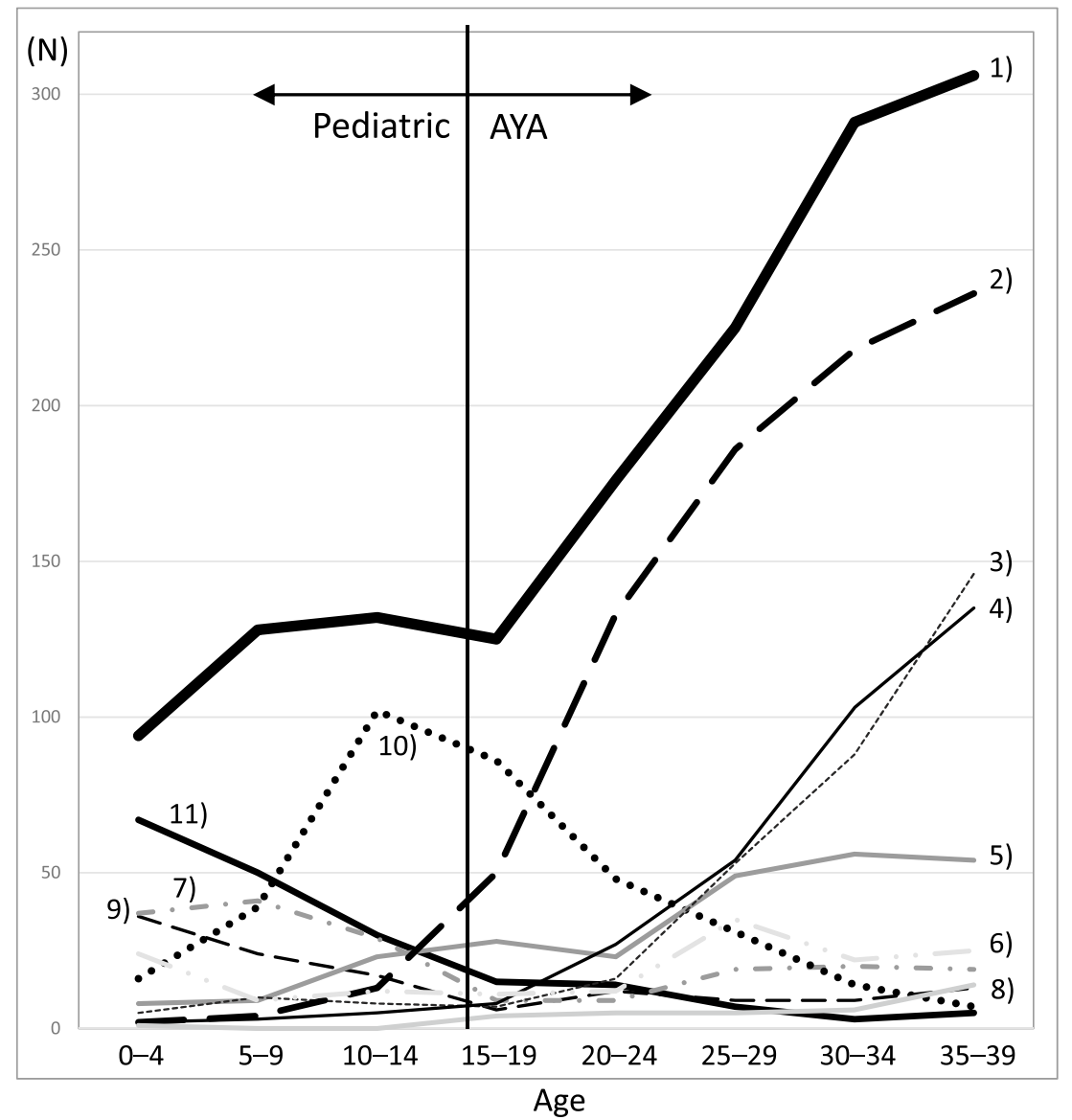

1) Gliomas

2) Pituitary adenomas

3) Meningiomas

4) Tumors of the cranial \& paraspinal nerve

5) Mesenchymal, nonmeningothelial tumors

6) Others (including chordoma, pineal parenchymal tumors, melanocytic tumors)

7) Tumors of the sellar region (e.g., craniopharyngioma)

8) Lymphomas \& hematopoeic tumors

9) Ependymal tumors

10) Germ cell tumors

11) Embryonal tumors

Fig. 1 Age-specific number of patients for each brain tumor at the brain tumor registry 2005-2008, Japan 
full facts: some desired full honest information to allow for autonomous choices; others preferred general information without details; and some wanted no bad news at all, only positive information [16]. The field of neurosurgery/ neuro-oncology is unique, because patients might experience brain tumor-specific problems (for instance, epilepsy, hemiparesis, and changes in cognition and personality). Cognitive changes may complicate the breaking of bad news. Information given about the prognosis should be tailored to the coping styles of individual patients and their relatives, rather than to the preference of individual clinicians or treatment centers [14]. Therefore, physicians need to collect information on the preferences of patients and their families in advance and then break the bad news gradually by grasping their comprehension.

\section{End-of-life care in AYA malignant brain tumors}

Advance care planning is an important element in improving end-of-life care, but malignant brain tumors remain underresearched [17]. Some malignant brain tumors in AYAs are incurable. Therefore, some AYA patients with malignant brain tumor are prematurely faced with mortality, and many do not discuss advance directives and living wills. In the final stage, patients with brain tumor present severe symptoms due to the tumor and/or its treatment, which require adequate palliative management and supportive therapy. Few physicians are equipped to explain the terminal nature of malignant brain tumors to AYAs, who, unconsulted, frequently die in hospitals [18-20]. AYAs may be forced by their parents to live with them without their spouse or children but against their will. The family preferences and patient's personal context should be considered before the patient is unable to express a living will, and appropriate psychological support should be provided. In incurable settings for AYAs, as with all patients, early palliative care and advanced care planning with end-of-life discussions are appropriate. Active intervention with sufficient visualized information benefited advance care planning for palliative care, avoiding cardiopulmonary resuscitation, and being confident in end-of-life decision making [21]. Therefore, the first step of breaking bad news is essential.

Dysphagia is an important symptom of patients with brain tumor, because it may affect nutrition and hydration, potentially inducing aspiration pneumonia [22]. In one study $85 \%$ of patients presented dysphagia in the last 4 weeks of life (median onset to death 21 days) [23]. Dysphagia also complicates the oral intake of drugs, including anticonvulsants. Seizures in the last 4 weeks before death occurred in 30\% of patients and repeated seizures or status epilepticus, in $6 \%$ of patients [23]. In the last weeks of life, patients presenting dysphagia require changes from oral to nasogastric intubation for anticonvulsants or intramuscular barbiturates. Some new antiepileptic drugs such as levetiracetam and lacosamide are administered intravenously.

End stage brain tumors often involve consciousness deterioration. Most patients with brain tumor are bedridden for longer than other patients with cancer because of consciousness deterioration and/or severe neurological deficit. Caregivers of large physique AYA patients encounter other challenges. Patients with brain tumor usually die peacefully, but delirium or behavioral disturbances may cause disruption [23]; agitation and restlessness with moaning and grimacing should be interpreted as physical pain. Bulbar palsy or pseudobulbar palsy might disrupt breathing, which may be eased via the nasopharyngeal airway. No-treatment end-of-life treatment decisions for AYA patients with brain tumor-including withdrawal of supportive treatment (steroid, anticonvulsant), withdrawing-withholding of artificial nutrition-hydration in patients in prolonged vegetative state, and palliative sedation - are the most difficult [17]. Advance directives can help in taking such decisions. AYA patients with brain tumor at the recurrent/progressive stage may not be able to decide if they are willing to live with severe neurological symptoms. Hence, earlier active intervention of advance care planning with sufficient information of patients and their families is essential. Early discussions with families and home care personnel are also important.

\section{Fertility preservation and malignant brain tumors}

Loss of fertility is a pitiful outcome of cancer treatment and has been reported to negatively impact the QOL of cancer survivors [24]. Currently, AYA patients with brain tumor are underserved because of poor prognosis and fear of treatment delay. Patients with primary brain tumors risk infertility via: (1) chemotherapy; (2) hypothalamic-pituitary hormonal malfunction through tumor infiltration, surgery, or radiotherapy; and (3) ovary exposure at cerebrospinal irradiation. Among these risks, chemotherapy could impair sperm production in men or deplete the pool of ovarian oocytes in women, hence becoming a target for fertility preservation [25]. Germinoma at neurohypophysis and craniopharyngioma are leading causes of hypothalamic-pituitary malfunction. Applying proton beam therapy for cerebrospinal irradiation avoids radiation exposure of thoracoabdominal organs including ovaries [26].

Due to the poor prognosis for AYA malignant glioma, fertility preservation is a well-recognized but infrequently addressed clinical issue, and only $30 \%$ of patients reported having a discussion about fertility preservation [27]. Temozolomide, a representative alkylating agent commonly used in the treatment of gliomas, impairs fertility [28]. Bevacizumab is also reportedly moderately toxic to ovaries [29]. Despite the poor prognosis, reproductive-particularly childless-AYA patients with malignant glioma have 
significant interest in fertility preservation [25]. However, female patients with glioma have another issue. Tumor progression during pregnancy has been reported in $33-45 \%$ of patients with glioma [30]. Another report showed that the growth rate of tumor during pregnancy increased in $87 \%$ of cases, while clinical deterioration was observed in $38 \%$ of cases. Clinical deterioration resolved after delivery in $21.4 \%$ of cases. Hence, it is important to inform female AYA patient with glioma and her partner about the impact of pregnancy on the growth of the glioma.

On the other hand, AYA medulloblastoma is a good candidate for fertility preservation, and NCCN guidelines advise not only fertility preservation but also contraception [31]. Newly diagnosed germinoma at the pineal lesion, usually developing only in males, is the best candidate for sperm cryopreservation. Germinoma at neurohypophysis-a poor candidate for fertility preservation - with equal incidence in both sexes, often decreases gonadotropin secretion. Although the efficacy of postchemotherapy testicular sperm extraction/intracytoplasmic sperm injection was reported recently, informing reproductive AYA males of the potential risk of treatment-induced infertility is important [32]. First line standard treatment in Japan of the three courses of carboplatin and etoposide is considered a moderate infertility risk. Sperm cryopreservation should be also considered for patients with recurrent germinoma, because second remission is possible via treatment with intensive chemotherapy and radiation; newly diagnosed germinoma involving neurohypophysis usually precludes sperm cryopreservation due to impaired gonadotrophs. Highly malignant germ cell tumors including choriocarcinoma, yolk sac tumor, and embryonal carcinoma challenge fertility preservation because of the rapid progression of these diseases.

Technical advances offer cryopreservation of ovarian tissue for not only reproductive age but also prepubertal girls at risk of sterility, and should be offered before initiation of chemotherapy [33]. The cryopreservation of ovarian tissue could instead be systematically offered even to prepubertal girls at risk of sterility due to gonadotoxic treatment. In 2019, the American Society for Reproductive Medicine reported that ovarian tissue cryopreservation and transplantation is no longer experimental, but a standard of care [34]. However, fertility preservation is more difficult for female patients, because they need to undergo extra surgical procedure. Patients and/or parents may decline such offers because of the poor prognosis for malignant brain tumors. Testicular tissue cryopreservation is still under investigation and future technical advancement is expected.

To address these unmet needs and improve the QOL of AYA brain tumor survivors, it is necessary to establish oncofertility programs, including universal counseling on individualized infertility risk assessment and available preservation options, at each institution. In Fig. 2, I

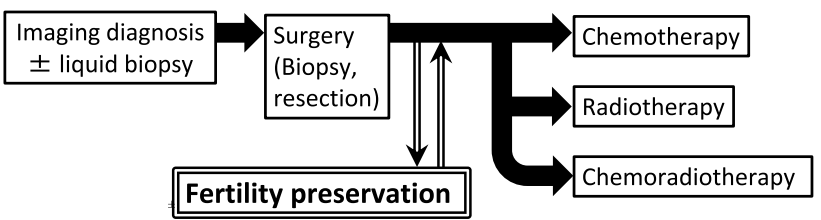

Fig. 2 Expected clinical course of patients with brain tumor at reproductive age

schematically show the representative clinical course of patients with brain tumor at reproductive age. An age-based guideline to the establishment of each disease and timely updates are essential.

\section{Educational supports}

AYAs, especially adolescent patients, require educational support during cancer treatment and for cancer survivors [8] - issues dependent on specific national education systems. The Japanese school system comprises 6-year elementary schools, 3-year junior high schools and 3-year senior high schools, followed by 2-or-3-year junior colleges or 4-year colleges. Compulsory education lasts for 9 years through elementary and junior high school. During compulsory education periods, schools for long-term treatment of in-patient children are usually available in cancer centers and university/medical university hospitals. Educational support for senior high school students with hematopoeic malignancy or solid cancer-including malignant brain tumors-is essential. However, the hospital class for senior high school is unavailable in most hospitals. Since 2017, Hiroshima University Hospital has joined the Board of Education in Hiroshima City/Prefecture to teach senior high school inpatients students [35]. Remote teaching using information and communication technology has been applied to patients since 2018; the use of avatar robot OriHime ${ }^{\circledR}$ has been developed and utilized for students at senior high school (Fig. 3). Remote lessons proved effective for continuing learning and communication with classmates and teacher, providing effective preparation for school life after discharge. The establishment of educational systems requires collaboration among hospitals, regional senior high schools, and the Board of Education in each prefecture. Under coronavirus disease 2019, remote classrooms are utilized for healthy students. Therefore, it is expected that the remote class become generalized for AYA patients during long-term hospitalization in the future.

Educational support for brain tumor survivors-including school reentry after discharge-presents challenges [12]. Malignant brain tumor survivors sometimes encounter difficulty at school reentry due to cognitive impairment, difficulties in concentration, memory, or problem-solving, as 


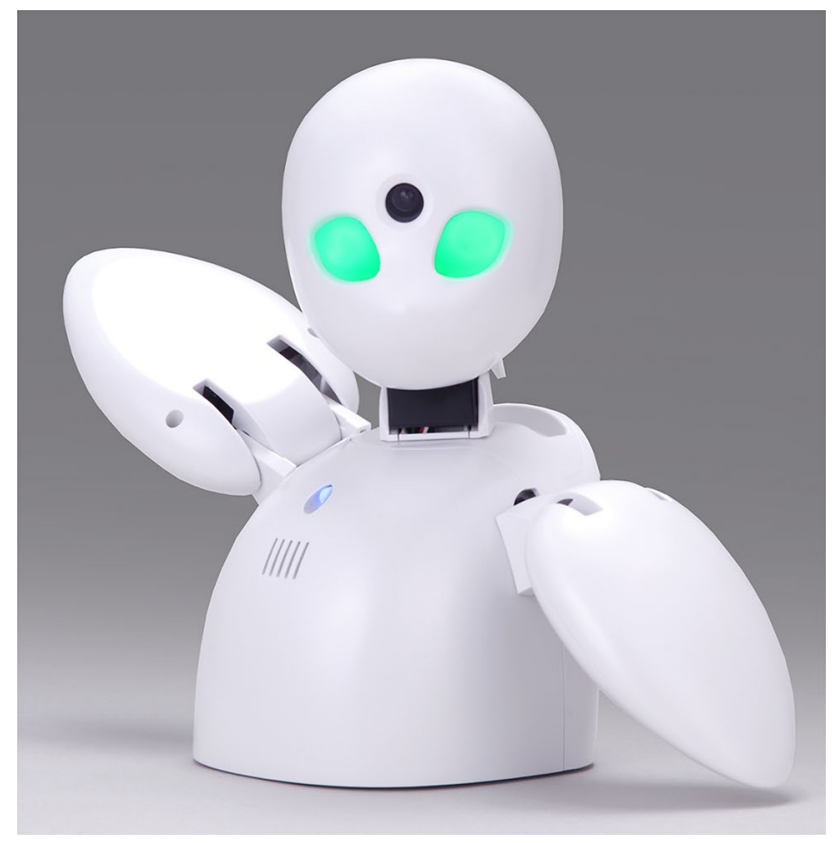

Fig. 3 Avatar robot OriHime ${ }^{\circledR}$ remote operation system

well as physical disabilities. Patients who underwent highdose cranial irradiation may suffer cognitive deterioration due to late effects of irradiation [36]. Brain tumor survivors also suffer from changes in appearance-including alopecia, sparse hair, short stature, obesity, and so on-which may result in negativity, demotivation, and anxiety. The most common side effect is fatigue [37, 38], from unknown causes, with sequelae including cognitive difficulties, hormonal deficiency, seizure and anticonvulsant use, anxiety and depression, and so on. After school reentry, teachers, while encouraging brain tumor survivors, should understand the problem of fatigue. Lack of understanding about the persistent fatigue of brain tumor survivors may be their biggest problem. Both predischarge coordination conferences and periodic coordination conferences with patients, care givers, social workers, hospital-school liaison, rehabilitation staff, nurses, and attending physicians may further understanding of brain tumor survivors' distress and alleviate their anxiety. Exact evaluation of their cognitive function, neuropsychological condition, and physical disabilities would provide support during compulsory education - but not during postcompulsory education. For AYA brain tumor survivors, assistance in utilizing social resources and peer support may be more useful. It was reported that a vast majority of AYAs remained engaged in education throughout their cancer trajectory with the support of educational support program [39]. Because AYA patients with brain tumor with cognitive impairment have many unmet needs, establishment of a special educational support program is desired. Further research will be required to evaluate the quality of interventions and incorporate the voice of AYA patients with brain tumors to further inform service delivery.

\section{Molecular biology and treatment of the AYA population in neuro-oncology}

Recent advances in molecular study have enhanced integrated diagnosis of brain tumors. For example, pediatric and adult molecular glioma subtypes differ greatly. In adult grade II and III diffuse gliomas, isocitrate dehydrogenase (IDH) $1 / 2$ and $1 p / 19 q$ codeletion status play an important role. Adults with diffuse gliomas harboring both IDH mutation and chromosome $1 \mathrm{p} / 19 \mathrm{q}$ codeletion show more favorable clinical outcomes with significantly longer survival. In contrast, adults with diffuse gliomas harboring IDH wildtype and $1 \mathrm{p} / 19 \mathrm{q}$ noncodeletion show poor clinical outcomes, which are consistent with glioblastoma. Adults with diffuse glioma harboring IDH mutation and 1p/19q noncodeletion show intermediate clinical outcomes [40]. IDH mutation and $\mathrm{O}^{6}$-methylguanine-DNA-methyltransferase methylation are highly linked, and confer more favorable responses to alkylator therapy—-specifically temozolomide [5]. In pediatric high-grade gliomas, histone 3 (H3) K27-alteredusually observed in pons and thalamic tumors-has a poor prognosis. In infant nonbrainstem glioma, $40 \%$ of tumors harbor NTRK-fusion [41]. In older child and adolescent hemispheric high-grade gliomas, H3 G34R/V mutation is common [7].

Low-grade gliomas of childhood may be single pathway diseases. In pilocytic astrocytoma-the most common low-grade glioma - activation of the RAS/MAPK pathway via BRAF/FGFR alteration has been reported [5]. Among BRAF/FGFR alteration, BRAF-KIAA 1549 fusion is most common and is associated with a better prognosis [42]. $\mathrm{BRAF}^{\mathrm{V} 600 \mathrm{E}}$ mutation, also observed in minor populations of pilocytic astrocytoma, was associated with a poorer prognosis. Mutation of $\mathrm{BRAF}^{\mathrm{V} 600 \mathrm{E}}$ was also identified, most commonly occurring in pleomorphic xanthoastrocytoma and ganglioglioma, followed by astroblastoma [43]. $\mathrm{BRAF}^{\mathrm{V} 600 \mathrm{E}}$ mutation may be associated with a better prognosis in some glioblastoma populations, but epithelioid glioblastoma with $\mathrm{BRAF}^{\mathrm{V} 600 \mathrm{E}}$ mutation showed a poorer prognosis [44]. In epithelioid glioblastoma, TERT promoter mutations and CDKN2A/B homozygous deletions were also reported [45].

The 5th edition of the WHO classification has taken a new approach to classifying gliomas, glioneuronal tumors, and neuronal tumors, dividing them into six different families as follows: (1) adult-type diffuse gliomas; (2) pediatrictype diffuse low-grade gliomas; (3) pediatric-type diffuse high-grade gliomas; (4) circumscribed astrocytic gliomas; (5) glioneuronal and neuronal tumors; and (6) ependymomas [7]. AYA gliomas comprise pediatric-type and adult-type. Molecular studies are essential for exact diagnosis. The 5th 
edition of the WHO classification will include much molecular information: CDKN2A/B homozygous deletion in diffuse astrocytoma IDH-mutant; MYB- or MYBL1-alteration in pediatric diffuse astrocytoma; ZFTA (the new designation for $\mathrm{C} 11$ orf95, which is considered more representative of the tumor type than RELA, because it may be fused with more partners than RELA) fusion supratentorial ependymoma; MNl-altered astroblastoma; CNS tumor with BCOR internal tandem duplication; CIC-rearranged sarcoma; and so on.

Importantly, special consideration is necessary for AYA brain tumors, because they occur at a developmentally sensitive period and AYAs are likely to survive long enough to experience their sequelae. Each indication of radiation therapy for brain tumors should take into account the risks of functional impairment caused by tumor progression versus the late effects of radiation, including: neurocognitive impairment, endocrine disorder, vascular events such as moyamoya syndrome, infraction and cavernous angioma, and increased risk of secondary malignancy [46, 47]. For example, physicians will recommend local irradiation for middle-aged adult patients with oligodendroglioma, but may try to avoid or postpone local irradiation for AYA patients with oligodendroglioma, a representative chemo- and radiosensitive adult-type diffuse glioma. On the other hand, physicians need to realize the difficulty of applying the standard pediatric regimen of vincristine and carboplatin against nonresectable AYA pilocytic astrocytoma due to the risk of gonadal toxicity and higher risk of vincristine neurotoxicity [5]. For some diseases, adults with pediatric tumors (such as medulloblastoma and germ cell tumors) seem to have a worse prognosis than is usually reported for children due to under treatment or erroneous treatment, or poor compliance with the therapeutic guidelines [48]. Adult patients' tolerance of chemotherapy following radiotherapy is generally lower and these treatments should, therefore, be applied at centers with experience in neuro-oncology. Survivorship of AYA patients with brain tumor is another issue. Future development of AYA glioma-specific treatment strategies is anticipated.

The vast majority of AYA patients with brain tumor appear to be spontaneous and unrelated to either carcinogens in the environment or hereditary predisposition, such as family cancer syndromes [4]. For AYA patients with brain tumor, current cancer multigene panel testing may be less useful for discovering therapeutic targets. However, cancer multigene panel testing would allow adolescent patients with brain tumor to reach an exact diagnosis, especially useful in differentiating between pediatric- and adult-type tumors. Accumulation of knowledge about AYA brain tumors using cancer multigene panel testing would enhance the development of integrated diagnosis and treatment. Future development with AYA brain tumor-specific cancer multigene panels would render development of precision medicine.
One important issue is the low participation rate of AYAs with cancer in clinical trials. A previous study reported that the participation rate of AYAs was 5-20\%, which was much lower than that of children (60-80\%) [49]. This low participation rate is associated with poor survival outcomes of AYAs. Improved survival has been documented among young adolescents with several tumor types who are treated according to the pediatric protocol as compared with older AYAs [50, 51]. Pediatric oncologists tend to be more aggressive in chemotherapy, whereas adult oncologists are more likely to choose radiation therapy. It is unclear whether this difference in the treatment approach changes the outcomes of AYA patients with brain tumors. Molecular targeted therapy should be developed via clinical trials, because this approach potentially reduces the late effect of chemotherapy/ radiotherapy. Cancer multigene panel testing in the diagnostic work-up of AYA brain tumors is critical for the development of molecular targeted therapy. An AYA neuro-oncology program should be developed in major cancer centers to improve the outcomes of AYA patients with brain tumors.

\section{Conclusions}

Here, I explained the current status and issues of AYA brain tumors. Although the incidence of entire AYA brain tumors is not rare, each pathologically based disease is rare and integrated diagnosis of AYA brain tumors is currently in transition. Because AYAs experience physical, psychological, emotional, social, and sexual change with many life events, specific consideration is necessary to prevent deteriorating QOL by brain tumor-specific symptoms. Although the prognosis for some malignant AYA brain tumors is poor, physicians must be cognizant that reproductive-and particularly, childless-AYA patients have significant interest in fertility preservation. All AYA brain tumors should undergo molecular profiling not only for diagnosis but also for investigating tumor biology and developing an appropriate treatment. Development of AYA brain tumor-specific treatment strategies and precision medicine are desirable to avoid sequelae and late effects and to improve outcomes.

\section{Declarations}

Conflict of interest The author declares that there is no conflict of interst.

\section{References}

1. Sender L, Zabokrtsky KB (2015) Adolescent and young adult patients with cancer: a milieu of unique features. Nat Rev Clin Oncol 12(8):465-480. https://doi.org/10.1038/nrclinonc.2015.92 
2. Katanoda K, Shibata A, Matsuda T et al (2017) Childhood, adolescent and young adult cancer incidence in Japan in 2009-2011. Jpn J Clin Oncol 47(8):762-771. https://doi.org/10.1093/jjco/ hyx070

3. Ostrom QT, Patil N, Cioffi G et al (2020) CBTRUS statistical report: primary brain and other central nervous system tumors diagnosed in the United States in 2013-2017. Neuro Oncol. https://doi.org/10.1093/neuonc/noaa200

4. Epelman S (2013) The adolescent and young adult with cancer: state of the art-brain tumor. Curr Oncol Rep 15(4):308-316. https://doi.org/10.1007/s11912-013-0329-1

5. Zapotocky M, Ramaswamy V, Lassaletta A et al (2018) Adolescents and young adults with brain tumors in the context of molecular advances in neuro-oncology. Pediatr Blood Cancer. https://doi.org/10.1002/pbc.26861

6. Close AG, Dreyzin A, Miller KD et al (2019) Adolescent and young adult oncology-past, present, and future. CA Cancer J Clin 69(6):485-496. https://doi.org/10.3322/caac.21585

7. Louis DN, Perry A, Wesseling P et al (2021) The 2021 WHO classification of tumors of the central nervous system: a summary. Neuro Oncol 23(8):1231-1251. https://doi.org/10.1093/neuonc/ noab106

8. Perales MA, Drake EK, Pemmaraju N et al (2016) Social media and the adolescent and young adult (AYA) patient with cancer. Curr Hematol Malig Rep 11(6):449-455. https://doi.org/10.1007/ s11899-016-0313-6

9. Jones JM, Fitch M, Bongard J et al (2020) The needs and experiences of post-treatment adolescent and young adult cancer survivors. J Clin Med. https://doi.org/10.3390/jcm9051444

10. Smith AW, Bellizzi KM, Keegan TH et al (2013) Health-related quality of life of adolescent and young adult patients with cancer in the United States: the Adolescent and Young Adult Health Outcomes and Patient Experience study. J Clin Oncol 31(17):21362145. https://doi.org/10.1200/JCO.2012.47.3173

11. Brain Tumor Registry of Japan (2005-2008) (2017). Neurol Med Chir (Tokyo) 57 (Suppl 1):9-102. doi:https://doi.org/10.2176/ nmc.sup.2017-0001

12. Zebrack B, Chesler MA, Kaplan S (2010) To foster healing among adolescents and young adults with cancer: what helps? What hurts? Support Care Cancer 18(1):131-135. https://doi.org/10. 1007/s00520-009-0719-y

13. Korsvold L, Lie HC, Mellblom AV et al (2016) Tailoring the delivery of cancer diagnosis to adolescent and young adult patients displaying strong emotions: an observational study of two cases. Int J Qual Stud Health Well-being 11:30763. https:// doi.org/10.3402/qhw.v11.30763

14. Davies E, Higginson IJ (2003) Communication, information and support for adults with malignant cerebral glioma : a systematic literature review. Support Care Cancer 11(1):21-29. https://doi. org/10.1007/s00520-002-0392-x

15. Goebel S, Mehdorn HM (2018) Breaking bad news to patients with intracranial tumors: the patients' perspective. World Neurosurg 118:e254-e262. https://doi.org/10.1016/j.wneu.2018.06.168

16. Malmstrom A, Akesson L, Milos P et al (2021) "Do I want to know it all?" A qualitative study of glioma patients' perspectives on receiving information about their diagnosis and prognosis. Support Care Cancer 29(6):3339-3346. https://doi.org/10.1007/ s00520-020-05846-7

17. Song K, Amatya B, Voutier C et al (2016) Advance care planning in patients with primary malignant brain tumors: a systematic review. Front Oncol 6:223. https://doi.org/10.3389/fonc.2016. 00223

18. Cohen-Gogo S, Marioni G, Laurent S et al (2011) End of life care in adolescents and young adults with cancer: experience of the adolescent unit of the Institut Gustave Roussy. Eur J Cancer 47(18):2735-2741. https://doi.org/10.1016/j.ejca.2011.09.008
19. Papageorgiou GI, Razis ED (2020) CNS tumors in adolescents and young adults: the need for a holistic specialized approach. JCO Oncol Pract 16(4):155-162. https://doi.org/10.1200/JOP. 18.00767

20. Sizoo EM, Pasman HR, Dirven L et al (2014) The end-oflife phase of high-grade glioma patients: a systematic review. Support Care Cancer 22(3):847-857. https://doi.org/10.1007/ s00520-013-2088-9

21. El-Jawahri A, Podgurski LM, Eichler AF et al (2010) Use of video to facilitate end-of-life discussions with patients with cancer: a randomized controlled trial. J Clin Oncol 28(2):305-310. https://doi.org/10.1200/JCO.2009.24.7502

22. Walbert T, Khan M (2014) End-of-life symptoms and care in patients with primary malignant brain tumors: a systematic literature review. J Neurooncol 117(2):217-224. https://doi.org/ 10.1007/s11060-014-1393-6

23. Pace A, Di Lorenzo C, Guariglia L et al (2009) End of life issues in brain tumor patients. J Neurooncol 91(1):39-43. https://doi. org/10.1007/s11060-008-9670-x

24. Loren AW (2015) Fertility issues in patients with hematologic malignancies. Hematology Am Soc Hematol Educ Program 2015:138-145. https://doi.org/10.1182/asheducation-2015.1. 138

25. Stone JB, Kelvin JF, DeAngelis LM (2017) Fertility preservation in primary brain tumor patients. Neurooncol Pract 4(1):40-45. https://doi.org/10.1093/nop/npw005

26. Lester-Coll NH, Morse CB, Zhai HA et al (2014) Preserving fertility in adolescent girls and young women requiring craniospinal irradiation: a case report and discussion of options to be considered prior to treatment. J Adolesc Young Adult Oncol 3(2):96-99. https://doi.org/10.1089/jayao.2013.0038

27. Stiner RK, Clarke JL, Sinha N et al (2019) Attitudes toward fertility and fertility preservation in women with glioma. Neurooncol Pract 6(3):218-225. https://doi.org/10.1093/nop/npy036

28. Strowd RE, Blackwood R, Brown M et al (2013) Impact of temozolomide on gonadal function in patients with primary malignant brain tumors. J Oncol Pharm Pract 19(4):321-327. https://doi.org/ $10.1177 / 1078155212469243$

29. Imai A, Ichigo S, Matsunami K et al (2017) Ovarian function following targeted anti-angiogenic therapy with bevacizumab. Mol Clin Oncol 6(6):807-810. https://doi.org/10.3892/mco.2017.1237

30. Tosoni A, Balestrini D, Brandes AA (2017) Fertility preservation in women with CNS tumors. Expert Rev Anticancer Ther 17(5):439-445. https://doi.org/10.1080/14737140.2017.1316195

31. Penas-Prado M, Theeler BJ, Cordeiro B et al (2020) Proceedings of the Comprehensive Oncology Network Evaluating Rare CNS Tumors (NCI-CONNECT) Adult Medulloblastoma Workshop. Neurooncol Adv 2 (1):vdaa097. doi:https://doi.org/10.1093/ noajnl/vdaa097

32. Kawai K, Nishiyama H (2019) Preservation of fertility of adult male cancer patients treated with chemotherapy. Int J Clin Oncol 24(1):34-40. https://doi.org/10.1007/s10147-018-1333-0

33. Wallace WH, Smith AG, Kelsey TW et al (2014) Fertility preservation for girls and young women with cancer: populationbased validation of criteria for ovarian tissue cryopreservation. Lancet Oncol 15(10):1129-1136. https://doi.org/10.1016/S14702045(14)70334-1

34. Practice Committee of the American Society for Reproductive Medicine. Electronic address aao (2019) Fertility preservation in patients undergoing gonadotoxic therapy or gonadectomy: a committee opinion. Fertil Steril 112(6):1022-1033. https://doi. org/10.1016/j.fertnstert.2019.09.013

35. Karakawa S, Inoue M, Sasaki S et al (2020) Using remote lessons for the effective support of senior high-school education for longterm inpatients at Hiroshima University Hospital. Jpn J Pediatric Hematol Oncol 57(4):182 
36. Gunn ME, Malila N, Lahdesmaki T et al (2015) Late new morbidity in survivors of adolescent and young-adulthood brain tumors in Finland: a registry-based study. Neuro Oncol 17(10):14121418. https://doi.org/10.1093/neuonc/nov115

37. Pace A, Dirven L, Koekkoek JAF et al (2017) European Association for Neuro-Oncology (EANO) guidelines for palliative care in adults with glioma. Lancet Oncol 18(6):e330-e340. https://doi. org/10.1016/S1470-2045(17)30345-5

38. Nicklin E, Velikova G, Hulme C et al (2019) Long-term issues and supportive care needs of adolescent and young adult childhood brain tumour survivors and their caregivers: a systematic review. Psychooncology 28(3):477-487. https://doi.org/10.1002/pon.4989

39. Dax V, Edwards N, Doidge O et al (2021) Evaluation of an educational and vocational service for adolescent and young adults with cancer: a retrospective review. J Adolesc Young Adult Oncol 10(1):56-65. https://doi.org/10.1089/jayao.2020.0009

40. Gonzalez Castro LN, Wesseling P (2021) The cIMPACT-NOW updates and their significance to current neuro-oncology practice. Neurooncol Pract 8(1):4-10. https://doi.org/10.1093/nop/npaa055

41. Wu G, Diaz AK, Paugh BS et al (2014) The genomic landscape of diffuse intrinsic pontine glioma and pediatric non-brainstem high-grade glioma. Nat Genet 46(5):444-450. https://doi.org/10. 1038/ng.2938

42. Collins VP, Jones DT, Giannini C (2015) Pilocytic astrocytoma: pathology, molecular mechanisms and markers. Acta Neuropathol 129(6):775-788. https://doi.org/10.1007/s00401-015-1410-7

43. Lehman NL, Hattab EM, Mobley BC et al (2017) Morphological and molecular features of astroblastoma, including BRAFV600E mutations, suggest an ontological relationship to other cortical-based gliomas of children and young adults. Neuro Oncol 19(1):31-42. https://doi.org/10.1093/neuonc/now118

44. Sugimoto K, Ideguchi M, Kimura T et al (2016) Epithelioid/ rhabdoid glioblastoma: a highly aggressive subtype of glioblastoma. Brain Tumor Pathol 33(2):137-146. https://doi.org/10.1007/ s10014-015-0243-3
45. Nakajima N, Nobusawa S, Nakata S et al (2018) BRAF V600E, TERT promoter mutations and CDKN2A/B homozygous deletions are frequent in epithelioid glioblastomas: a histological and molecular analysis focusing on intratumoral heterogeneity. Brain Pathol 28(5):663-673. https://doi.org/10.1111/bpa.12572

46. Yamasaki F, Takayasu T, Nosaka R et al (2017) Development of cystic malacia after high-dose cranial irradiation of pediatric CNS tumors in long-term follow-up. Childs Nerv Syst 33(6):957-964. https://doi.org/10.1007/s00381-017-3400-7

47. Vatner RE, Niemierko A, Misra M et al (2018) Endocrine deficiency as a function of radiation dose to the hypothalamus and pituitary in pediatric and young adult patients with brain tumors. $\mathrm{J}$ Clin Oncol 36(28):2854-2862. https://doi.org/10.1200/JCO.2018. 78.1492

48. Mascarin M, Coassin E, Franceschi E et al (2019) Medulloblastoma and central nervous system germ cell tumors in adults: is pediatric experience applicable? Childs Nerv Syst 35(12):22792287. https://doi.org/10.1007/s00381-019-04340-8

49. Yeo KK, Burgers DE, Brodigan K et al (2021) Adolescent and young adult neuro-oncology: a comprehensive review. Neurooncol Pract 8(3):236-246. https://doi.org/10.1093/nop/npab001

50. Albritton KH, Wiggins CH, Nelson HE et al (2007) Site of oncologic specialty care for older adolescents in Utah. J Clin Oncol 25(29):4616-4621. https://doi.org/10.1200/JCO.2006.08.4103

51. Margol AS, Yeo KK, Xia C et al (2018) A comparative analysis of clinicopathological features and survival among early adolescents/ young adults and children with low-grade glioma: a report from the Children's Oncology Group. J Neurooncol 140(3):575-582. https://doi.org/10.1007/s11060-018-2983-5

Publisher's Note Springer Nature remains neutral with regard to jurisdictional claims in published maps and institutional affiliations. 